\title{
Mortality after emergency department intubation
}

\author{
Leon D. Sanchez • J. Scott Goudie • \\ Jennifer De la Pena • Kevin Ban • Jonathan Fisher
}

Received: 25 January 2008 / Accepted: 26 March 2008 /Published online: 31 May 2008

(C) Springer-Verlag London Ltd 2008

\begin{abstract}
Introduction The purpose of this study is to identify the rate of emergency department (ED) intubation and the mortality associated with ED intubation.

Methods We conducted a retrospective chart review of all patients intubated in the ED between 1 January 2004 and 31 December 2004 at an urban level one trauma centre with approximately 50,000 ED visits annually. All ED intubations were identified and reviewed. Two investigators reviewed all charts and collected the following data: age, sex, and final disposition from hospital as well as reason for intubation. The main outcome measure was survival to hospital discharge.

Results One hundred sixty-three intubations were reviewed. Of the total 163 patients, 44 (27.0\%) died prior to discharge from the hospital, 42 (25.8.\%) patients were discharged to a skilled nursing facility (e.g. nursing home, rehabilitation and extended care facility) and $71(43.6 \%)$ patients were discharged home. Dividing our cohort into trauma and nontrauma subgroups, $38 / 126(30.2 \%)$ of the non-trauma patients and $6 / 37(16.2 . \%)$ of the trauma patients died. The mean age for all patients in our study group was 61.5 years, with trauma patients being younger than the non-trauma subgroup. The mean age for trauma patients was 50.1 while non-trauma patients had a mean age of 64.8 .
\end{abstract}

Disclaimer: The views expressed in this paper are those of the author(s) and not those of the editors, editorial board or publisher.

L. D. Sanchez $(\bowtie) \cdot J$. S. Goudie · J. De la Pena $\cdot$ K. Ban •

J. Fisher

Department of Emergency Medicine,

Beth Israel Deaconess Medical Center,

One Deaconess Road WCC-2,

Boston, MA 02215, USA

e-mail: leon_sanchez@bidmc.harvard.edu
Conclusions The mortality after an ED intubation in our study population was relatively high. Further studies need to confirm these findings and help identify predictors of mortality.

Keywords Airway Intubation · Mortality . Emergency department

\section{Introduction}

The ability to mange the airway of critically ill patients is a defining skill in the practice of emergency medicine. Several studies comparing emergency physicians' (EP) skills at intubation with other practitioners, primarily anaesthesiologists, found similar proficiency $[1,2]$. Complications of emergency department (ED) intubations have been studied, but most only examine the peri-intubation period. While there are several studies that provide data as to rates of success of ED intubation and need for cricothyrotomy, there is little information as to the long-term prognosis of these patients.

Patients who are intubated in the ED are some of the most critically ill patients in the ED; the mortality after ED intubation has not been well studied. Previous studies have shown that the lay public have a lack of understanding of the dismal outcomes associated with cardiac arrests [3]. Understanding the outcomes of ED intubation can be useful in discussion with families of patients as well as provide information for future research. The purpose of this study is to identify the rate of ED intubation and the mortality associated with ED intubation.

\section{Materials and methods}

We conducted a retrospective chart review for all ED intubations performed over a 1-year period between 1 
Table 1 Age and mortality of patients intubated in the ED

\begin{tabular}{llll}
\hline & Overall & Trauma & Non-trauma \\
\hline$N$ & 163 & 37 & 126 \\
Age & $61.3(58.0-64.7)$ & $49.0(40.5-57.5)$ & $64.5(61.7-68.3)$ \\
Mortality & $27.0 \%$ & $16.2 \%$ & $30.2 \%$ \\
& $(20.0-33.9)$ & $(4.1-28.3)$ & $(22.2-38.3)$ \\
\hline
\end{tabular}

January 2004 and 31 December 2004 at an urban level one trauma centre with approximately 50,000 ED visits annually. ED intubations were identified retrospectively by reviewing the hospital financial databases for intubations for all ED patients. Patients that had critical care billing for an intubation procedure or for ventilator use in the ED were obtained. ED patient logs were also examined to identify all patients who were admitted to the intensive care unit (ICU), the operating rooms or died. The charts were reviewed to determine if the intubation took place in the ED. All identified intubations occurring in the ED were included in the analysis. Patients who were intubated prior to arrival in our ED either by another hospital or by prehospital personnel were excluded from our cohort. Two investigators reviewed all charts and abstracted the following data: age, sex, final disposition from hospital, reason for intubation using a standardized list of terms and trauma versus non-trauma. If all data were not available from the discharge summary, the original chart was reviewed to gather the necessary data. De-identified data were entered into a Microsoft Excel 2003 (Redmond, WA, USA) database and analysed using Stata V8.2 (College Station, TX, USA). The main outcome measure was survival to hospital discharge. Confidence intervals were calculated and comparisons were made using unpaired $t$-tests and Fisher's exact tests. The study was approved by the Institutional Review Board.

\section{Results}

One hundred and sixty-three patients were intubated in our ED during the 1-year study period. This is approximately 3 intubations for every 1,000 patients seen. Of the total 163 patients, $44(27.0 \%)$ died prior to discharge from the hospital, and $72(44.2 \%)$ patients were discharged to home or left against medical advice (AMA) (1 patient). Forty-two (25.8.\%) patients were discharged to a skilled nursing facility (e.g. nursing home, rehabilitation and extended care facility) for further care. Dividing this patient population into trauma and non-trauma subgroups, $6 / 37(16.2 \%)$ of the trauma patients died and 38/136 (30.2\%) of the non-trauma patients died. Of the trauma patients who died, $5 / 6$ had intracranial haemorrhage (ICH). The mean age for all patients was 61.3 years, with trauma patients being younger (49.0 versus 65.0 ) than the non-trauma subgroup. The mean age of the survivors was lower than the non-survivors: 57.3 versus $72.2(P<0.0001)$. No children were identified as having been intubated in our study, as our institution does not routinely care for paediatric patients. See Tables 1 and 2 for a complete summary of the data.

Of the deaths, $38.6 \%$ occurred within $24 \mathrm{~h}$ of intubation. An additional 22.7, 29.5 and 9.1\% of deaths occurred at $72 \mathrm{~h}$, 7 days and 30 days, respectively, after intubation. No deaths were found to occur beyond 30 days of hospitalization.

\section{Discussion}

In our search of the literature no large studies reporting mortality after intubation in the ED were found. The NEAR database, the largest ED airway registry at present, provides insight into the ED course of these patients, but does not collect post-intubation data or outcomes on their patients [4]. Most of the emergency medicine literature concentrates on the mechanics of the intubation and the time surrounding the event. Considerable emergency medicine research focuses on the tools of airway assessment and techniques to improve intubation success such as the LEMON score and the BURP maneuver [5, 6]. Alternatively, there is a lack of information on how these patients do once they leave the $\mathrm{ED}$, and how the various techniques impact on the mortality associated with ED intubation. While the patients who require ED intubation certainly represent a sicker population, our study demonstrates a relatively high mortality rate. Now that we know the mortality rate of $27 \%$, we can begin to examine those interventions that not only improve the success of ED intubation, but also ultimately improve mortality.

As an initial step, we chose to do a retrospective chart review of all ED intubations for a 1-year period. While recognizing the limitations of such an approach, we thought this initial study would provide some useful information and indications for future research. We chose survival to hospital discharge as our primary endpoint. The considerable mortality rate following intubation is not surprising as the need for intubation in the ED probably represents a marker for severity of disease. Like mortality from sepsis or

Table 2 Comparison of age of intubated patients survived vs died

\begin{tabular}{llll}
\hline & All & Trauma & Non-trauma \\
\hline $\begin{array}{l}\text { Survivor } \\
\text { (mean age) }\end{array}$ & $57.3(53.3-61.3)$ & $44.0(34.9-53.0)$ & $62.0(58.1-66.0)$ \\
$\begin{array}{c}\text { Death } \\
\text { (mean age) }\end{array}$ & $72.2(67.4-76.9)$ & $74.8(65.6-84.0)$ & $71.7(66.4-77.1)$ \\
\hline
\end{tabular}


after a myocardial infarction, establishing a baseline mortality rate after ED intubation will provide us with an outcome measure we can use to test the benefit of specific interventions.

Due to the limited number of patients in our cohort, many questions remain unanswered. Stratification of the patients by disease entity could not be achieved in a meaningful way. Stratification by disease entity also presents the inherent problem of classification. For example, should head injuries be classified as neurological or as traumatic? The one major subdivision of patients we could perform analysis on was trauma versus non-trauma patients. There was lower mortality in the trauma patients. This finding could be explained by the lower age of trauma patients and their ability to respond to medical interventions. In both groups, older patients had a higher mortality. This is most evident in the trauma group where the mean age was 44.0 years, but the mean age of those who died was 74.8 years as compared to the non-trauma group. This is an expected result. Older patients with myocardial infarction and stroke have been demonstrated to have worse outcomes in the literature [7,8]. Older patients tend to have less physiological reserve so the same insult would be expected to have a greater deleterious effect upon prognosis [9].

Yanagawa et al., in a study comparing intubation without medications versus with medications for non-traumatic $\mathrm{ICH}$, reported the mortality of the two groups as 46.8 and $72.9 \%$, respectively [10]. The study had 70 patients for their control and study group combined and the main outcome measure was the Glasgow Outcome Score, not mortality. If we select non-traumatic ICH from our patients, 9/16 (56.2\%) died. The high mortality rate in the ICH subgroup, and the differences in the trauma and non-trauma groups, suggest that with a large enough number of patients subgroup analysis may reveal differences in mortality by specific disease entity.

Survival to hospital discharge was chosen over 30-day mortality since it eliminated the problem of having patients that could not be followed up while still providing a meaningful endpoint.

\section{Limitations}

This study was conducted as a pilot study to provide background data as to what directions would be of interest in future studies. As a single-centre retrospective study we are limited in our ability to make general statements based on our study population. As we saw no paediatric patients, we cannot make any inferences as to their mortality rate after intubation. The small sample size limits our ability to provide subgroup analysis for outcomes based on the reason for intubation other than the fairly broad trauma/ non-trauma division. Since there is no central way to identify patients who were intubated in our ED, we used multiple methods to identify cases; however, there is a small chance we still could have overlooked a particular group that could alter the outcome.

\section{Conclusion}

The mortality after an ED intubation in our study population was $27.0 \%$. Future studies should attempt to identify predictors of mortality among ED intubations as well as look at this question in a multicentre setting. Prospective data collection would help gather data in a more reliable manner.

\section{References}

1. Levitan RM, Rosenblatt B, Meiner EM, Reilly PM, Hollander JE (2004) Alternating day emergency medicine and anesthesia resident responsibility for management of the trauma airway: a study of laryngoscopy performance and intubation success. Ann Emerg Med 43:48-53

2. Bushra JS, McNeil B, Wald DA, Schwell A, Karras DJ (2004) A comparison of trauma intubations managed by anesthesiologists and emergency physicians. Acad Emerg Med 11:66-70

3. Van den Bilck JJ (2002) The impact of television fiction on public expectations of survival following inhospital cardiopulmonary resuscitation by medical professionals. Eur J Emerg Med 9: 325-329

4. Sagarin MJ, Barton ED, Chang YM, Walls RM (2005) Airway management by US and Canadian emergency medicine residents: a multicenter analysis of more than 6,000 endotracheal intubation attempts. Ann Emerg Med 46:328-336

5. Reed MJ, Dunn MJG, McKeown DW (2005) Can an airway assessment score predict difficulty at intubation in the emergency department? Emerg Med J 22:99-102

6. Knill RL (1993) Difficult laryngoscopy made easy with a "BURP". Can J Anaesth 40:279-282

7. Schuler J, Maier B, Behrens S, Thimme W (2006) Present treatment of acute myocardial infarction in patients over 75 years - data from the Berlin Myocardial Infarction Registry (BHIR). Clin Res Cardiol 95:360-367

8. Engelter ST, Reichhart M, Sekoranja L, Georgiadis D, Baumann A et al (2005) Thrombolysis in stroke patients aged 80 years and older: Swiss survey of IV thrombolysis. Neurology 65:1795-1798

9. Alter DA, Manuel DG, Gunraj N, Anderson G, Naylor CD, Laupacis A (2004) Age, risk-benefit trade-offs, and the projected effects of evidence-based therapies. Am J Med 116:540-545

10. Yanagawa Y, Sakamoto T, Okada Y, Tuzuki N, Katoh H et al (2005) Intubation without premedication may worsen outcome for unconsciousness patients with intracranial hemorrhage. Am J Emerg Med 23:182-185 